\title{
Case Study: Challenges in building and maturing an internet connection in rural mineral mining companies in South
}

\section{Africa}

\author{
DP du Plessis \\ Tshwane University of Technology
}

\begin{abstract}
Connecting any company's Local Access Network (LAN) to a Wide Area Network (WAN) or the internet should be relatively easy because there are technologies available that enable companies to connect seamlessly. However, in the rural parts of South Africa are some geographical and economical challenges when companies in rural areas want to connect their LAN to a WAN or the internet with the biggest challenges being the availability of technology and the affordability of technology for these small rural companies. This paper discusses these challenges experienced and proposes a framework that can be followed by these rural companies when connecting a LAN to a WAN or the internet.
\end{abstract}

Keywords: Wide Area Network, Local Access Network, Rural ICT Network, Mining, ICT4D, ICT for Developing Country

\section{Introduction}

Three mining companies in South Africa were used for this research project. Mining plays a significant role in the South Africa economic. Smit (2013) states that mining is responsible for $20 \%$ of the GDP of the South African economy. Mines are predominantly situated in rural areas (underdeveloped areas) where data networks are not readily available while head offices of these mines are situated in the city (developed areas). Some of these rural mining companies are small companies with small budgets for information technology but simply need a connection to the internet. Parker et al (1995) states that several studies concluded that a positive relationship exists between access to telecommunications capabilities in rural areas and improvement in certain economic indicators. Without adequate connections to advanced telecommunications infrastructure 
and services, rural communities may not be able to fully participate in the emerging information economy.

Mining companies used for this research project are very much dependent on two kinds of technologies; mining technology and Information technology. Mining technology is the different tools and equipment used for mining processes, while information technology includes ICT systems and ICT networks (hardware and software). ICT systems are dependent on ICT networks to insert, extract and distribute data. For these ICT systems to be accessible and well equipped for the necessary workload, a reliable and well-designed network is necessary to provide for the required data transfers. Research methodology used for this study was Case Studies as well as Action Research and the next section will explain the application of these two methodologies as part of this research project.

\section{Methods}

As laid out in the precious section, the research problem of the study was about connecting the LAN of rural mining companies, to a WAN or the internet, using the limited technology available in these rural areas. Figure 1 below gives a schematic representation of the LAN, WAN and internet connected to each other.

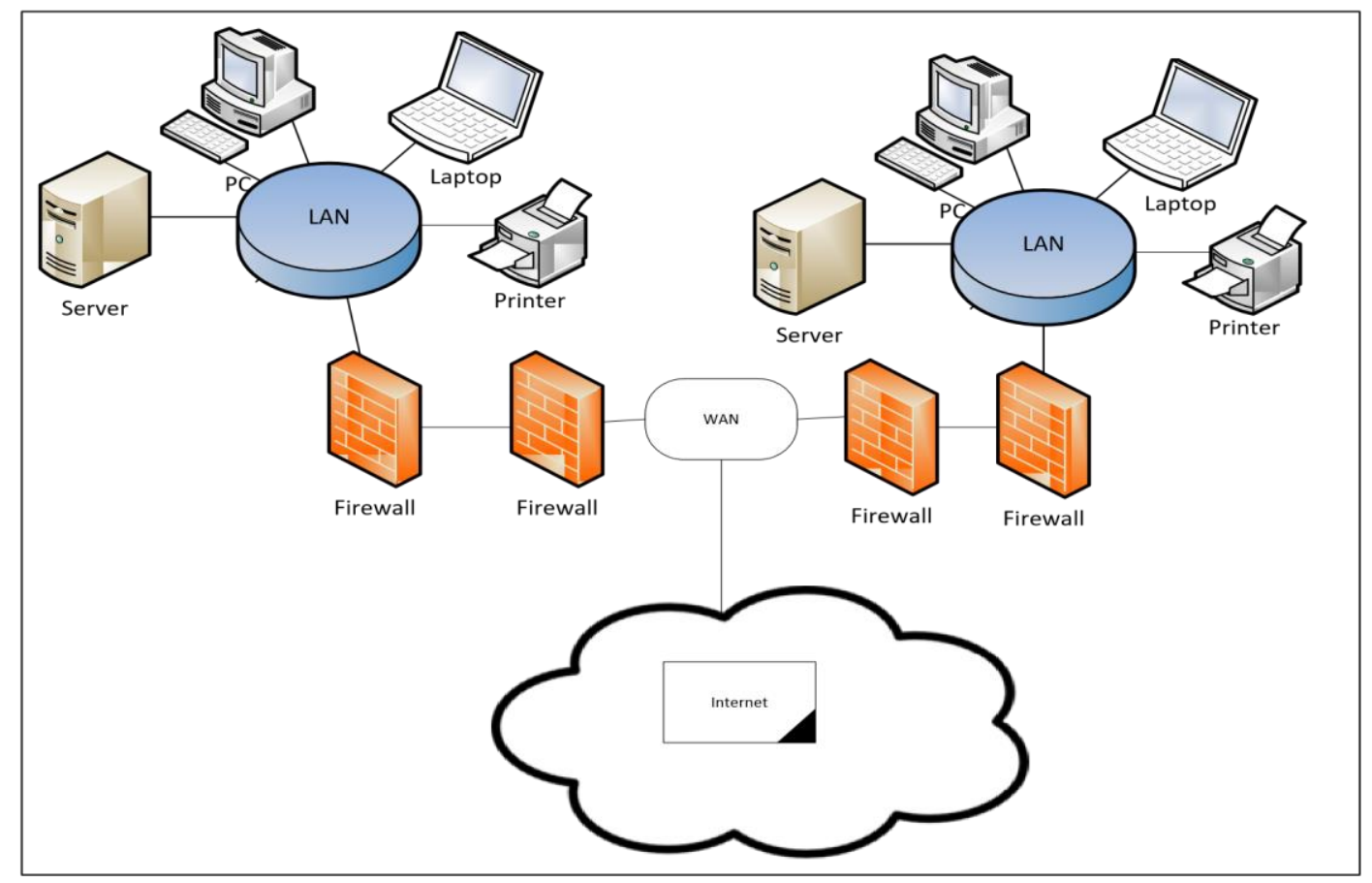

Figure 1: Two LANs connected to a WAN and the internet 
In addressing this problem, the study was guided by the following research questions and sub questions:

1. What technologies are available in these rural areas?

2. What criteria can be used to select a solution from available technologies?

a. Are these technologies cost effective when considering the installation and maintenance of it?

b. What possible downtime can occur because of remoteness?

3. How can selected technologies optimally be used to connect these companies to a WAN or the internet?

4. How can the LAN and WAN be designed to minimize bandwidth consumption?

With regard to the study type, this was a case study (Yin, 2003) combined with action research (Avison et al., 2008; Oates, 2006; Chiasson et al., 2009). The goal of action research is to solve a practical problem in some organization, while engaging a theoretical reflection, and eventually delivering theoretically significant results.

Blum (1955) discussed two stages in action research - a diagnostic stage followed by a therapeutic stage. During the diagnostic stage the researcher studies the social situation. The therapeutic stage involves the change of experiments up to a stage where it satisfies the hypothesis. Changes are designed and introduced, and the results are studied to introduce more changes to improve the situation. Susman and Evered's (1978) approach is in accord with these prescripts albeit more analytical. They defined five phases in action research (refer to Baskerville, 1999). These are:

(1) diagnosing,

(2) action planning,

(3) action taking,

(4) evaluating and (5) specifying learning.

The first phase is about identifying the problem to solve. In this study it is about the challenges to connect the LAN of a rural company to a WAN or the internet and optimizes the design of the WAN to accommodate low bandwidth usage. The second to fifth phases are addressing the problem based on a theoretical framework. Please see figure 2 below:

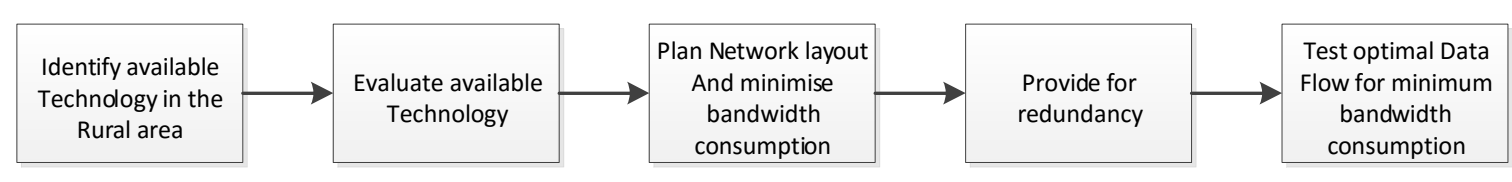


Figure 2: Framework for rural WAN or Internet connectivity

The framework for rural WAN or Internet connectivity consists of five processes that will be discussed in sections 3, 4 and 5 below.

\subsection{Case study}

Oates (2008) believes that a case study tests and investigates the real life situation. Case studies are used in this research project to address the geographical challenges of rural South Africa when connecting a LAN to a WAN or internet. Yin (2003) defined a case study as "an empirical inquiry that investigates a contemporary phenomenon within its real-life context, especially when the boundaries between phenomenon and context are not clearly evident." ICT networks are normally studied with the focus on solving technical problems with a minimum of geographical challenges associated with it. The focus of this study is to highlight the challenges of the geographical areas and the availability of suppliers of the required technology in these areas which made the boundaries between phenomenon and context difficult to discover.

Implementing these technologies could differ from the implementation of the same technology in developed areas because of the following reasons:

- Distance from developed areas for maintenance of technologies.

- Theft of cables and power supply equipment installed in distant areas.

- Rental cost of the diginet line as one of the available technologies. Rental is also charged per kilometre from the closest Internet Service Provider (ISP) connection.

- Distributed information system user base, where users are situated on the same LAN than the information system, while others need to use the internet links and connect via a Virtual Private Network (VPN) to these information systems. This connection uses the internet connection.

\subsection{Action research}

In order to address all these practical challenges, action research was used as an integral part of the case studies. The researcher was actively involved in the planning and implementation of the solution. Baskerville (1999) described action research as an established research method used in the social and medical sciences since the midtwentieth century. Towards the end of the 1990s its use began growing in popularity 
in scholarly investigations of ICT. Chiasson et al. (2009) states that action research is a research method that develops a solution whilst solving a practical problem. New knowledge is created during this process (Chiasson et al. 2009).

The section below describes the first process of the framework for rural WAN or internet connectivity.

\section{Availability of Technology for WAN or Internet connectivity}

\subsection{Asymmetric Digital Subscriber Line (ADSL)}

Three mines in the rural areas of the Northern Cape in South Africa were used in this study. ISPs in this rural area do not have Asymmetric Digital Subscriber Line (ADSL) as a product. There are on the other hand not a lot of companies that render an internet connection services in the rural areas and suppliers in these areas don't find some of the products economically viable because of a lack of interest of variety customers in products like ADSL.

\subsection{Radio Link}

Other technologies available, like radio links, are more expensive to implement and maintained because of the remoteness of the area. Radio links need a number of repeater sites with available power supply at all the repeater sites. Installation of these repeater sites do not only require construction projects but the maintenance of these repeater sites requires traveling of long distances which can result into high traveling cost and long network downtime periods.

\subsection{Very Small Aperture Terminal (VSAT)}

A Very Small Aperture Terminal (VSAT) is a two-way satellite, using dish antennas on surface to interface between two service networks. Data transfer rates range from 4 kilo bites per second up to 4 mega bites per second; however, there are modules available that can handle a rate of up to 16 mega bites per second on VSAT. VSATs access satellites in geosynchronous orbit to relay data from small remote earth stations (terminals) to other terminals (in mesh topology) or master earth station "hubs" (in star topology). Suppliers of these services use real-time monitoring 24 hours a day 7 days a week. Payment for this service consists of a fixed monthly fee.

\subsection{Diginet}

Diginet is a Private Leased Circuit (PLC) and normally run from point to point. The speed of this line is guaranteed. It is seen as one of the most reliable way of connecting Virtual Private network (VPN) branches and carrying voice traffic. Suppliers of these services use real-time monitoring 24 hours a day 7 days a week. Payment for this service consists of a fixed monthly fee. The maximum speed available for diginet is 2 mega bites 
per second. That means that 120 mega bites per minute that is the maximum data that can be transfer in one direction. In our project the transfer was needed in two directions, and therefore the transfer rate in one direction goes down to 60 mega bites per minute. When a breakdown appears in this rural area, it takes a maximum of 48 hours or longer to repair because the supplier of diginet is available in rural areas and start reparations immediately.

\subsection{Design of the solution}

The mining companies used for this research only used diginet and VSAT to connect their LAN's to the internet. Diginet was used as the default solution with an automatic switch over to VSAT as the redundant solution. These two technologies are the only technologies currently available and affordable in the area. Linking the LAN of these mining companies to a WAN or the internet can be very expensive. Considering the limited budget for ICT it was important for these companies to plan their ICT network properly so that they can achieve more from available technology. Section 4 below explains the network design to be followed to optimally utilize existing technologies and avoid unnecessary ICT expenses.

\section{Plan network layout and minimize bandwidth consumption}

Mining companies with these challenges are therefore required to follow a network design strategy that provide for these challenges. The two available technologies, mentioned above, are also expensive and when considering the lower commodity prices world-wide since early 2015, mining companies are looking for opportunities to save money. It is therefore important to optimize resources instead of growing it.

The other challenge is that the company needs communication networks to manage the business while also communicating with all stakeholders like suppliers, shareholders, customers, government to mention a few.

Diginet was used as the default solution with VSAT as a backup (redundant) solution. VSAT was used as the back-up solution because VSAT maintain a signal latency of approximately 500 milliseconds and the cost of VSAT is almost double the cost of diginet when in use permanently. The 500 milliseconds signal latency cause a lot of frustration to the users when used for services like video conferencing. Die backup solution was needed because the turnaround time for Diginet was 48 hours.

To optimise these internet connections the following was done: 


\section{Network design}

5.1 Set quality of service using QOS Techniques like Classification and Marking, Congestion, Policing and Shaping, Avoidance and Queuing on the routing switch with the following traffic priorities:

- $\quad$ Priority 1: Video traffic

It is important that there is no distortion on the video that will make communication in these meetings difficult.

- $\quad$ Priority 2: Voice Over IP (VOIP) traffic if used.

We did not used VOIP as part of the projects because the telephone system was connected straight to landline operator and therefore no bandwidth was needed for VOIP.

- $\quad$ Priority 3: Hyper Text Transfer Protocol (HTTP) traffic.

oWhen serving the internet, people expect to get a response in seconds otherwise they complain that they experience slow responses. Internet usage was monitored and unnecessary traffic was removed. Analysis was done on the firewall to determine the internet domains that are generating the highest amount of traffic. This exercise resulted in all social media domains been blocked which was creating a lot of additional traffic because of large number of images been downloaded.

oThe home page of the web browsers was changed with a domain group policy on Active Directory (Domain controller) on all domain users to start in an intranet web page which doesn't need to download any data via the internet link. The supplier of the web browser normally creates the default homepage settings to, by default, open their internet website. That means that every time a user opens the browser, it automatically opens this internet site which generates additional http traffic which is using limited resources (bandwidth).

oWork related domains were identified and internet users were limited on the firewall to these work related sites. Work related domains are the domains on the internet that users need to access to do their job.

- $\quad$ Priority 4: Simple Mail Transfer Protocol (SMTP) traffic o Set attachment size limits on the email system. This limit user to send big attachments on email that is taking a lot of bandwidth.

o Audit high email users to limit private emails and educate users.

o The email spam filter was moved from the company's LAN (local) to the Internet Service Provider (ISP) to stop the spam before it takes up bandwidth on the internet line. 
- $\quad$ File Transfer Protocol (FTP) o FTP was blocked on the firewall and was given to users on request based on the required merits.

5.2 Decentralize infrastructure with servers situated at the office where the majority of users are sitting with reporting system (data warehouse) at the central point for end to end reporting. Updates are run after hours to avoid extra traffic during the day. Reporting information is therefore one day old. If newer information is needed an extra run could be done manually in the middle of the day for a specific data request.

\section{Test optimal data flow and minimize bandwidth consumption}

The possible data flow has been analyzed to determine the optimal way of data transfer. Data that was transferred over the WAN was identified.

Next two steps in action research are about making a change happen and then assessing it. In our study the following was changed and assessed to minimize data traffic on the internet link (WAN).

- $\quad$ Servers were moved to the LAN where the biggest user-group was situated.

- Quality of service was used to give a priority to some data packages above

others. With email data users normally do not have a problem if it is delayed for a couple of minutes.

The fifth step in action research refers to norming what is learned to serve as a guide for organizational practice. At the time of our study, this step was initiated; a time limitation prevented us from seeing its completion. Data analysis methods included system requirements collection and keeping a diary of observation. These methods provided data that were subjected to content analysis and used for analysing observation records, and creating system design documentation.

\section{Conclusion}

Implementing and maturing an internet connection for a rural companies requires that the project team not only look at technical aspect of the technology, but it is important to look at the geographical conditions and how best the connection can be design to address these geographical challenges. The biggest impact of a rural area in South Africa is the fact that new technology is not always available in rural areas and potential user of these technologies has to use older technology while designing there network to achieve maximum results. 


\section{References}

Avison, D., Dwivedi, Y.K., Fitzgerald, G. and Powell, P., "The beginnings of a new Era: Time to reflect on 17 years of the ISJ," Information Systems Journal, Vol. 18, No. 1:5-21, 2008.

Baskerville, R.L. 1999. Investigating information systems with action research. Communications of the AIS, 2(19): 1-133.

Blum, F.H. 1955. Action research: a scientific approach? Philosophy of Science, 22(1): $1-7$.

Chiasson, M., Germonprel, M. \& Malhiassen, L. 2009. Pluralist action research: a review of the information systems literature. Information System Journal, 19(1): 31-54.

Oates, B.J. 2008. Researching information systems and computing. London: Sage. 341 p.

Parker, E., Hudson, H., Dillman, D., Strover, S., \& Williams, F. 1995. Electronic byways: State policies for rural development through telecommunications. Washington: The Aspen Institute. p 325

Smit, C. 2013. The role of mining in South African Economy. http://www.sablog.kpmg.co.za/2013/12/role-mining-south-african-economy/

Strover, S. 2001. Rural internet connectivity. Telecommunication Policy Volume 25. Issue 5. Elsevier Science Ltd. P 331-347

Susman, G. and Evered, R.D. 1978 Action research. [Online]. Available: http://www.opuscollege.net/docs/appropriate_development/files/assets/downloa ds/page0120.pdf, Date of access: 08 DEC 2016.

Yin, R.K. 2003. Case study research: design and methods. 3rd ed. Thousand Oaks, CA: Sage. 